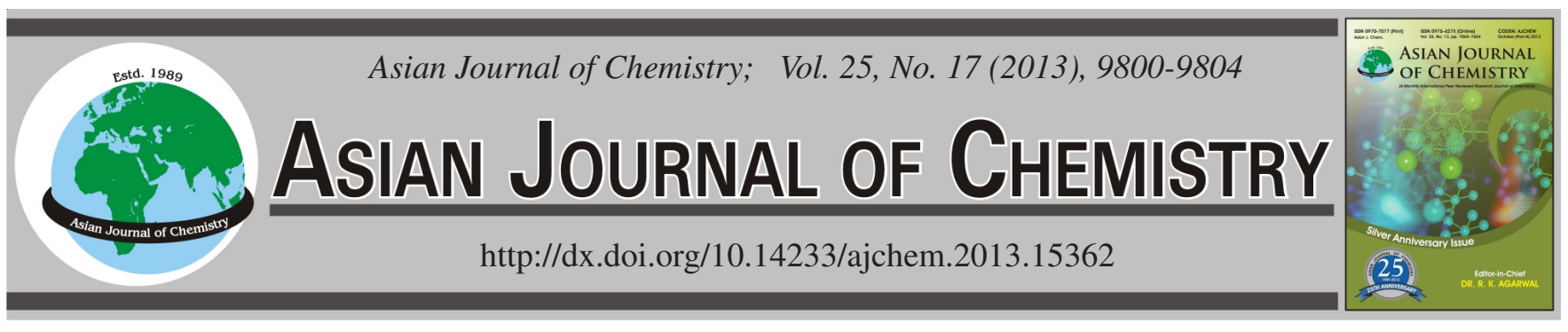

\title{
Antioxidant, Antimicrobial Potential and Phytochemical Attributes of Impatiens edgeworthii
}

\author{
Muhammad Mushtaq ${ }^{1}$, Nadeem Anwer $^{2, *}$, Muhammad Asim Waqar ${ }^{1}$, \\ Shabanem LatiF ${ }^{3}$, Shaukat Ali Shahid ${ }^{4}$ and Adeela Azam ${ }^{2}$
}

\begin{abstract}
${ }^{1}$ Department of Chemistry and Biochemistry, University of Agriculture, Faisalabad-38040, Pakistan
${ }^{2}$ Department of Chemistry, Government College University, Lahore-54000, Pakistan

${ }^{3}$ Department of Pharmacy, Euro Campus Hajvery University, Lahore-54000, Pakistan

${ }^{4}$ Department of Physics, University of Agriculture, Faisalabad-38040, Pakistan
\end{abstract}

*Corresponding author: E-mail: anwargcu@gmail.com

(Received: 22 February 2013;

Accepted: 23 October 2013)

AJC-14278

In this work, we investigated the availability of antioxidant, antimicrobial and phytochemical agents native to Impatiens edgeworthii in
various extraction solvents. The fractional extracts obtained for different solvents showed substantial levels total phenolic $(124-430 \mathrm{mg} / \mathrm{g}$
$\mathrm{GAE}$ ), anti-radical potential $(32-89 \%)$ and total antioxidant capacity $0.3-0.8$ versus gallic acid and butylated hydroxy toluene having
total antioxidant capacity value $0.85-1.2$. Phytochemical analysis confirmed the presence of flavonoids, sugars, alkaloids and saponins. in
vitro disc diffusion assay showed that all the extracts of $I$. edgeworthii have the antimicrobial activity comparable with standard antibiotics.
Furthermore, among tested solvents ( $n$-hexane, chloroform, ethyl acetate and methanol) ethyl acetate was found to be more effective for
the recovery of phytochemicals, phenolics, antioxidants and antimicrobial agents. The results of present study indicate $I$. edgeworthii is
latent source of potential bioactive and might be further explored for the development of chemopreventive drugs and nutraceuticals.

Key Words: Effective extraction, Phytochemicals, Antioxidants, Impatiens edgeworthii.

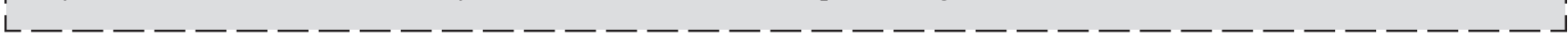

\section{INTRODUCTION}

Plants produce diverse potent bioactive during different catabolic and metabolic functions. The presence of these metabolites not only made plants to protect them from certain environmental factors but also medicinal source throughout human history. The colour, texture, odor and taste are also influenced by the nature and amount of these plant bioactives. It has been widely accepted that indigenous medicinal uses of plants, especially in rural areas are ascribed to the presence of phytochemicals and other bioactives ${ }^{1-3}$.

In the last few years, plants are most extensively studied materials for the exploration and development of nutracueticals and chemo preventive against degenerative diseases. A large amount of pharmaceuticals, antioxidants, antibacterials, antifungals and pesticides have been isolated, characterized and administered to living systems. Plant based phytochemicals and nutracuetical scavenge reactive oxygen species (ROS), reactive nitrogen species (RNS) and reactive glucose species (RGS) implicated in the pathology of a vast variety of diseases including cancer, atherosclerosis, diabetic mellitus, hypertension, AIDS and aging ${ }^{4-6}$.

Impatiens edgeworthii Hook. f. (Balsaminaceae) indigenous to Margallah Hills, Pakistan blossoms under shady areas and 1800-3000 $\mathrm{m}$ high open places ${ }^{7}$. The genus Impatiens is rich in organic acids, anthraquinones and flavonoids. Charles and Hagen ${ }^{8}$ have reported the isolation of three monoglucosides of kaempferol, quercetin and pelargonidin from the stem of I. balsamina. Similarly salicylic acid, sinnapic acid, caffeic acid, scopletin, 2-hydroxy, 1,4-naphthoquinone and 2-methoxy1,4-naphthoquinone had been extracted and purified from the stem of I. balsamina ${ }^{9}$. Panichayupakaranaut ${ }^{10}$ isolated a new biscoumarin, 4,40-biisofraxidin, from the roots of I. balsamina. The extracts of I. biflora and I. bicolor are documented as potential source of antioxidants, antibacterial and antifungals $^{11,12}$. To our best of knowledge no reports are available regarding the phytochemical composition and biological activities of I. edgeworthii. So the considered work was planned to explore phytochemicals, phenolics, antioxidants and antimicrobial agents native to I. edgeworthii. It is well established that the availability of plant bioactive depends upon the nature of target material and solvent applied for extraction. For this, a variety of solvents with variable polarity was practiced to check their extractability.

\section{EXPERIMENTAL}

The plant I. edgeworthii was collected from Ayubia Park, Muree and was identified at the Department of Botany, GC 
University, Lahore. The collected samples were washed with distilled water, dried at ambient and stored in air tight polythene bags after grinding.

The reagents and standards including gallic acid, trichloroacetic acid, linoleic acid, butylated hydroxy toluene (99.0\%) (BHT), Folin Ciocalteu reagent, sodium nitrite, ammonium thiocyanate, ferric chloride, ferrous chloride, potassium fericyanate, dimethyl sulfoxide, iodine and 2,2-diphenyl-1picryl-1-hydrazyl were purchased from Sigma Aldrich (St, Louis, MO, USA) (USA), while anhydrous sodium carbonate, chloroform, methanol, ethyl acetate, $n$-butanol and $n$-hexane were of analytical grade (Merck, Germany) whereas microbial culture strains and media were purchased from Oxoid (UK).

Extraction: The fractionation of the bioactive native to ground to I. edgeworthii was accomplished by extracting $20 \mathrm{~g}$ ground sample with $n$-hexane, chloroform, ethyl acetate and methanol, sequentially based on increasing polarity. The crude extracts obtained for $n$-hexane (ENH), chloroform (ECL), ethyl acetate (EEA) and methanol (EME) were filtered and concentrated at reduced temperature using rotary evaporator (EYELA, SB-651, Rikakikai Co. Ltd. Tokyo, Japan).

Phytochemical analysis: The obtained extracts of $I$. edgeworthii (ENH, ECL, EEA and EME) were tested for the presence of different phytochemicals using the tests as described by Sofowara ${ }^{13}$. Categorically, tannins were determined by dissolving $0.5 \mathrm{~mL}$ of each extracts in $1 \mathrm{~mL}$ of water and 1-2 drops of ferric chloride. Appearance of blue and green black colouration indicated gallic tannins and catecholic tannins, respectivley ${ }^{14}$. Saponins were visualized by foam test as follows; $1 \mathrm{~mL}$ of extract was shaken with small amount of water and the presence of foam until $10 \mathrm{~min}$ revealed the presence of saponin. Flavonoids were tested by alkaline Reagent test as; extracts were treated with few drops of sodium hydroxide solution and the formation of intense yellow colour which became colourless on addition of dilute acid indicated the presence of flavonoids ${ }^{15}$. For steroids, $2 \mathrm{~mL}$ of acetic anhydride was added to $0.5 \mathrm{~mL}$ extract along with $2 \mathrm{~mL} \mathrm{H}_{2} \mathrm{SO}_{4}$. The colour changed from violet to blue or green indicated the presence of steroids. Alkaloids were measured by Mayer's reagents. The alcoholic extract was evaporated to dryness and the residue was heated on a boiling water bath with $2 \%$ hydrochloric acid. After cooling, the mixture was filtered and treated with a few drops of Mayer's reagent. The samples were then observed for the presence of turbidity or yellow precipitation $^{16}$.

DPPH radical scavenging assay: The antiradical potential of extracted fractions I. edgeworthii: ENH, ECL, EEA and EME in tested solvents was evaluated at different concentration using the method of Lee et al. ${ }^{17}$. Briefly, $1 \mathrm{~mL}$ each extracts at different concentrations 25, 50, 100, 200, 300 and $400 \mu \mathrm{g} / \mathrm{mL}$ in methanol was mixed with $2 \mathrm{~mL}$ of methanolic solution of DPPH (10 mg/L). The mixture was shaken vigorously and allowed to stand at room temperature for $5 \mathrm{~min}$ followed by absorbance at $517 \mathrm{~nm}$ against methanol as the blank in a spectrophotometer. Lower absorbance of reaction mixture indicated higher free radical scavenging activity. The per cent of $\mathrm{DPPH}^{\bullet}$ discoloration of the sample was calculated according to the formula.

$$
\text { Antiradical activity }=\left(1-\frac{\mathrm{A}_{\text {Sample }}}{\mathrm{A}_{\text {Control }}}\right) \times 100
$$

$\mathrm{A}_{\text {control }}=$ Absorbance of $\mathrm{DPPH}^{\bullet}$ solution (containing all the reagents except test sample. $\mathrm{A}_{\text {sample }}=$ Absorbance of $\mathrm{DPPH}^{*}$ solution, 5 min after adding the extract.

Total antioxidant capacity (TAC): Total antioxidant activities of extracts were evaluated by phosophomolybdenum through complex formation method ${ }^{17}$. For this purpose, $0.1 \mathrm{~mL}$ solution of extracts and standards (gallic acid and BHT) in methanol $(0.5 \mathrm{mg} / \mathrm{mL})$ were combined with $1.9 \mathrm{~mL}$ of reagent solution $\left(0.6 \mathrm{M} \mathrm{H}_{2} \mathrm{SO}_{4}, 28 \mathrm{mM}\right.$ sodium phosphate and $4 \mathrm{mM}$ ammonium molybdate). The blank solution containing $2 \mathrm{~mL}$ reagent was used as control. The vials were capped, incubated in a water bath at $95^{\circ} \mathrm{C}$ and absorbance was measured at 695 $\mathrm{nm}$ at different time intervals after cooling the samples to room temperature. The total antioxidant capacity (TAC) of extracts of I. edgeworthii: ENH, ECL, EEA and EME determined in comparison with gallic acid (GA) and butylated hydroxy toluence (BHT).

Determination of total phenols: Total phenolic content of different solvent extracts were determined by using FolinCiocalteu reagent ${ }^{18}$ using gallic acid as standard. Briefly, $0.1 \mathrm{~mL}$ of different solvent extracts was combined with, 2.8 $\mathrm{mL}$ of $10 \% \mathrm{Na}_{2} \mathrm{CO}_{3}$ and $0.1 \mathrm{~mL}$ of Folin-Ciocalteu reagent. After $40 \mathrm{~min}$ absorbance was measured at $725 \mathrm{~nm}$ by using UV-visible spectrophotometer. Total phenolic were determined as mg equivalent of gallic acid per gram of dry extract by using the calibration equation below

$$
\mathrm{X}=\mathrm{Y}-\frac{0.00973}{0.0087}\left(\mathrm{R}^{2}=0.9973\right)
$$

where $\mathrm{X}$ and $\mathrm{Y}$ indicate absorbance and concentration respectively.

Antimicrobial activity: All the fractions (ENH, ECL, EEA and EME) of I. edgeworthii were individually tested against different fungal and microbial strains including; Proteus micabilus, Salmonella typhimorium, Bacillus subtillus, Bacillus lichaniform and Escherichia coli using disc diffusion assay ${ }^{19}$. Nutrient agar (Oxoid, UK) $28 \mathrm{~g} / \mathrm{L}$ was suspended in distilled water, mixed well and distributed homogenously. The medium was sterilized by autoclaving at $121^{\circ} \mathrm{C}$ for $15 \mathrm{~min}$. Before the medium was transferred to Petri plates; inoculum $(100 \mu \mathrm{L} / 100 \mathrm{~mL})$ was added to the medium and poured in sterilized petri plates. Now, small filter paper discs were laid flat on growth medium containing $100 \mu \mathrm{L}$ of extracts. The petri plates were then incubated at $37^{\circ} \mathrm{C}$ for bacteria and $30{ }^{\circ} \mathrm{C}$ for fungus for $24 \mathrm{~h}$. The extracts having antibacterial activity, inhibited the bacterial growth and clear zones were formed after $24 \mathrm{~h}$ which were measured in mm using zone reader. Ciprofloxacin and fluconazole were used as control against bacteria and fungus, respectively.

Statistical analysis: All the experiments were run in triplicate and data was reported and mean \pm SD. The extracts obtained by solvent fractions were analyzed by one way analysis of variance (ANOVA) using Statistica (version 8.1. Stat soft Inc, Tulsa Okahoma, USA) at $95 \%$ confidence interval. 


\section{RESULTS AND DISCUSSION}

Phytochemical composition: The presence of phytochemicals in different fractions of I. edgeworthii is documented Table-1. The phenols, sugar, flavonoids, saponins and triterpenoids were found to be present in all extracts whereas alkaloids were available in ethyl acetate and methanol extracts. No tannins were detected in any fractions of I. edgeworthii. It is revealed that $I$. edgeworthii contains diverse range of phytochemicals and availability of these phytochemicals varies with the polarity of the solvent applied for extraction. In considered work we observed that most of the phytochemical were detected in ethyl acetate fraction (EEA) of I. edgeworthii followed by methanol (EME), $n$-hexane (ENH) and chloroform (ECL). Phytochemicals are well known due to their medicinal, antimicrobial and antioxidant activity ${ }^{20}$. Flavonoids have been reported to possess antibacterial, antioxidant, antiinflammatory, antiallergic, antimutagenic and vasodilatory activities. Saponins showed hypocholesterolemic and antidiabetic properties, while steroids are well known due to analgesic properties ${ }^{18,21}$. The presence of biologically important phytochemicals in $I$. edgeworthii extracts may contribute to the medicinal value and chemo preventive potential of I. edgeworthii.

Total phenolic content: The total phenolic contents of fractions of I. edgeworthii estimated using Folin-Ciocalteu versus gallic acid as standard reference were 124.2-429.4 mg gallic acid equivalent GAE/g of dry extract. The higher amount of phenolic components available in ethyl acetate fraction (EEA) (Fig. 1) indicated the optimum polarity for the extraction of phenolic compounds from I. edgeworthii. The results were also supported by phytochemical analysis data obtained during the study.

The higher antiradical capacity and total antioxidant capacity (TAC) of EEA fraction are ascribed to the increased extractability of EEA fraction. The overall phenolic extraction capacity of the solvent fractions were descended as EEA > EME $>$ ECL $>$ ENH. Further the phenolic contents determined (124-429 mg GAE/g) during the present work were comparable with those observed in our previous study on Impatiens bicolor $^{12}$ exposing 104-532 mg GAE/g of phenolics and methanol as efficient solvent.

Antioxidant activity: A great number of aromatic, spicy, medicinal and other plants contain chemical compounds that exhibit antioxidant properties. Numerous studies were carried out on some of these plants, which resulted in the development of natural antioxidant formulations for food, cosmetic and other applications ${ }^{22}$. The antioxidant activity of extract fractions ENH, ECL, EEA and EME of I. edgeworthii was ranged $0.3-0.8$ as compared to gallic acid (1.2) and BHT (0.87) as shown in Fig. 1. Fig. 1 indicates that the ethyl acetate fraction of I. edgeworthii has total antioxidant capacity (TAC) comparable with standard antioxidants BHT and gallic acid. The higher antioxidant of activity of ethyl acetate fraction was also supported by the results of phytochemical analysis revealing the higher availability of plant bioactive for the said solvent. The overall order of total antioxidant capacity among tested solvent fractions of I. edgeworthii was observed as EEA $>\mathrm{EME}>\mathrm{ECL}>\mathrm{ENH}$.

TABLE-1

PHYTOCHEMICAL PROFILE OF DIFFERENT FRACTIONS OF Impatiens edgiwortthi

\begin{tabular}{|c|c|c|c|c|c|c|}
\hline \multirow{2}{*}{$\begin{array}{c}\text { S. } \\
\text { No. }\end{array}$} & \multirow{2}{*}{ Target bioactive } & \multirow{2}{*}{ Reagent applied } & \multicolumn{4}{|c|}{ Fractions of I. edgiwortthi } \\
\hline & & & $n$-Hexane & Ethyl acetate & Chloroform & Methanol \\
\hline 1 & Phenols & $\mathrm{FeCl}_{3}$ & + & ++ & ++ & ++ \\
\hline 2 & Sugar & Urea-HCl & H & ++ & ++ & ++ \\
\hline 3 & Flavonoids & Lead acetate & + & ++ & ++ & ++ \\
\hline 4 & Alkaloids & Dragondroffs & - & ++ & - & ++ \\
\hline 5 & Alkaloids & Cobalt thiocyanate & - & ++ & - & ++ \\
\hline 6 & Tannins & Butanol-HCl & - & - & - & - \\
\hline 7 & Saponins and triterpenoids & Ceric sulphate & + & ++ & ++ & ++ \\
\hline 8 & Flavonoids & $1 \% \mathrm{AlCl}_{3}$ & H & ++ & ++ & ++ \\
\hline 9 & Flavonoids & Benedict test & + & ++ & + & ++ \\
\hline 10 & Alkaloids & Iodine/KI & - & ++ & + & ++ \\
\hline
\end{tabular}
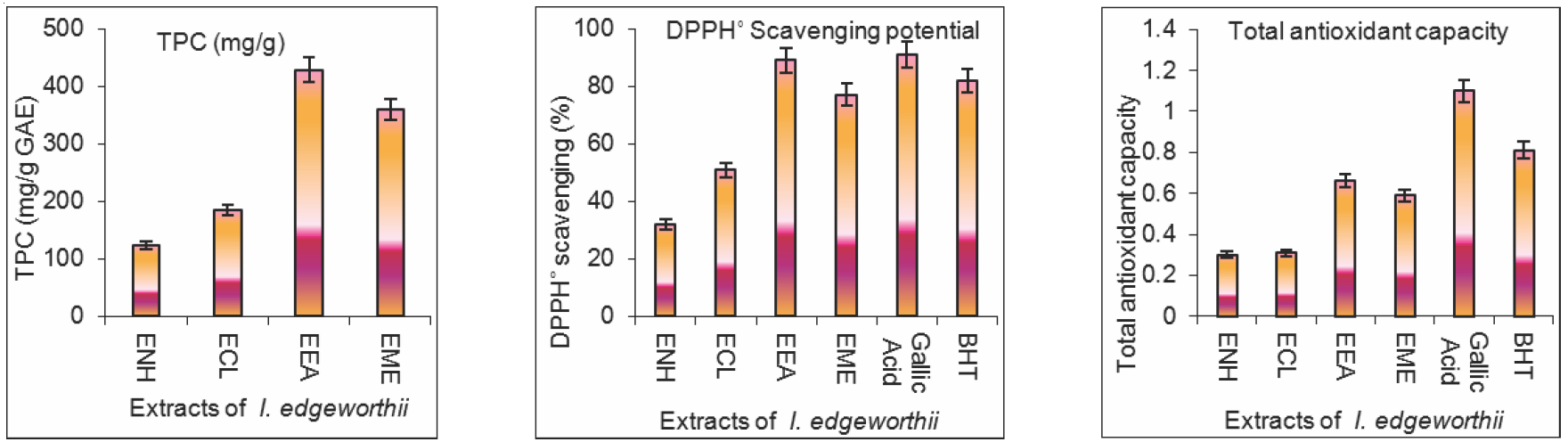

Fig. 1. Total phenolic (mg/g GAE), DPPH scavenging (\%) and total antioxidant activity of extracts of I. edgeworthii 
The antioxidant capacity assessed in terms of $\mathrm{DPPH}^{\bullet}$ scavenging ability of the extracts has been successfully used to estimate free radical-scavenging activity. Antioxidants neutralize DPPH radical by donating hydrogen or electron ${ }^{10}$. The DPPH radical scavenging activity of different fractions of I. edgeworthii ranged from 3 to $92 \%$ at different concentrations of extract as documented in Table-2. The results regarding the $\mathrm{DPPH}^{*}$ scavenging activity of different fractions investigated varied significantly $(p<0.05)$ as indicated by different alphabets in superscripts.

Among the solvents fractions practiced, ethyl acetate fraction (EEA) was found to be more efficient $\mathrm{DPPH}^{\bullet}$ scavenger as compared to methanol (EME), chloroform (ECL) and $n$-hexane (ENH) extracts. The ethyl acetate fraction of $I$. edgeworthii showed significantly high 63.61, 58.75, 52.14 and $45.91 \%$ DPPH radical scavenging activity at 400, 300, 200, 100,50 and $25 \mu \mathrm{g} / \mathrm{mL}$, respectively which might be ascribed to the higher availability of phytochemical and plant bioactive in ethyl acetate.

Antimicrobial activity: During last few decades, interest has been increased in plant antimicrobials to control pathogens and/or toxin producing microorganisms. Antibiotics provide the main basis for the therapy of bacterial infections. However, the high genetic variability of bacteria enables them to rapidly evade the action of antibiotics by developing antibiotic resistance. Thus there has been a continuing search for new and more potent antibiotics and in this context we evaluated different fractions I. edgeworthii against set of bacterial and fungal strains.

The antimicrobial activities of different solvent fractions of I. edgeworthii plant were tested against P. micabilus, $S$. typhimorium, B. subtillus, B. lichaniform and E. coli using disc diffusion assay ${ }^{19}$. The antibacterial activity [inhibition zone $(\mathrm{mm})$ diameter around test disc] of selected bacterial strains was determined in comparison with ciprofloxacin, while antifungal activity versus fluconazole. Ethyl acetate fraction of I. edgeworthii (EEA) showed significant antifungal and antibacterial activity against $P$. micabilus $(9 \mathrm{~mm})$, S. typhimorium (7 mm), B. subtillus $(12 \mathrm{~mm})$ and $E$. coli $(10 \mathrm{~mm})$ followed by methanol (EME), chloroform (ECL) and $n$-hexane (ENH) fraction as given in Table-3. According to Moghadam et al. ${ }^{23}$ the antimicrobial activity of medicinal plant material would beprobably due to the presence of alkaloids, flavonoides such as harmine/harmadine/harmadol in these compounds or
TABLE-3

ANTIMICROBIAL ACTIVITY OF DIFFERENT FRACTIONS (100 g/mL) I. edgeworthii IN COMPARISON WITH $10 \mu \mathrm{g} / \mathrm{mL}$ OF CIPROFLOXACIN AND FLUCONAZOLE

\begin{tabular}{lcccc}
\multicolumn{1}{c}{ Extract bicolor } & Hexane & Chloroform & $\begin{array}{c}\text { Ethyl } \\
\text { acetate }\end{array}$ & Methanol \\
\hline P. micabilus & 6 & 8 & 9 & 8 \\
S. typhimorium & 10 & 8 & 7 & 9 \\
B. subtillus & 5 & 9 & 12 & 11 \\
B. lichaniformis & - & - & - & - \\
E. coli & 2 & 5 & 10 & 6 \\
\hline In
\end{tabular}

Inhibition zone $(\mathrm{mm})$ diameter around test disc.

most probably soluble in organic polar solvent. So, the higher antimicrobial activity of ethyl acetate fraction of I. edgeworthii indicated the higher availability of phytochemicals and phenolic bioactives to this fraction. This trend in antimicrobial activity was also supported by total phenolic contents, antiradical potential against DPPH radicals and total antioxidant capacity.

\section{Conclusion}

This study explored the availability of diverse phytochemicals, phenolics, antioxidants and antimicrobial components of I. edgeworthii and results obtained in this regard were quite interesting. Among solvents used in this study, ethyl acetate was observed to be most effective for the recovery of biological attributes. It was concluded that the I. edgeworthii plant extracts have a high phytochemical contents, quite good free radical scavenging and antioxidant capacity as well as ample antibacterial and antifungal activities. The extracts of I. edgeworthii in different solvents can be used as an antibacterial and antifungal agent practically against the microorganism used during present study. The results regarding phytochemicals, phenolics, antioxidant and antimicrobial attributes indigenous to $I$. edgeworthii plant suggests that it can be used for the development of functional foods, antibiotics and nutraceuticals.

\section{ACKNOWLEDGEMENTS}

The authors thank Dr. Muhammad Shahid, Department of Chemistry and Biochemistry, University of Agriculture, Faisalabad, for providing technical assistance during the research work.

\begin{tabular}{|c|c|c|c|c|c|c|c|c|c|}
\hline \multirow{3}{*}{ S. No. } & \multirow{3}{*}{$\begin{array}{l}\text { Conc. } \\
(\mu \mathrm{g} / \mathrm{mL})\end{array}$} & \multicolumn{6}{|c|}{$\begin{array}{c}\text { TABLE-2 } \\
\text { DPPH RADICAL SCAVENGING ACTIVITY OF EXTRACTS OF } \text { I. edgeworthii }\end{array}$} & & \\
\hline & & \multicolumn{2}{|c|}{ ENH } & \multicolumn{2}{|c|}{ ECL } & \multicolumn{2}{|c|}{ EME } & \multicolumn{2}{|c|}{ EEA } \\
\hline & & Abs & Percentage & Abs & Percentage & Abs & Percentage & Abs & Percentage \\
\hline 1 & 400 & 0.173 & $32.5^{\mathrm{a}}$ & 0.170 & $33.7^{\mathrm{a}}$ & 0.085 & $66.9^{\text {cd }}$ & 0.074 & $71.01^{\mathrm{d}}$ \\
\hline 2 & 300 & 0.179 & $29.5^{\mathrm{a}}$ & 0.175 & $31.9^{\mathrm{a}}$ & 0.098 & $61.87^{\mathrm{d}}$ & 0.094 & $63.61^{\mathrm{d}}$ \\
\hline 3 & 200 & 0.195 & $24.1^{\mathrm{a}}$ & 0.200 & $22.17^{\mathrm{a}}$ & 0.118 & $54.1^{\mathrm{d}}$ & 0.106 & $58.75^{\mathrm{d}}$ \\
\hline 4 & 100 & 0.210 & $18.2^{\mathrm{b}}$ & 0.225 & $12.45^{\mathrm{a}}$ & 0.143 & $44.35^{\mathrm{c}}$ & 0.123 & $52.14^{\mathrm{d}}$ \\
\hline 5 & 50 & 0.248 & $3.5^{\mathrm{a}}$ & 0.245 & $4.6^{\mathrm{a}}$ & 0.169 & $34.24^{\mathrm{c}}$ & 0.139 & $45.91^{\mathrm{d}}$ \\
\hline 6 & 25 & 0.256 & $0.3^{\mathrm{a}}$ & 0.254 & $1.16^{\mathrm{a}}$ & 0.188 & $26.48^{\mathrm{c}}$ & 0.155 & $39.68^{d}$ \\
\hline 7 & Gallic acid & 0.020 & 92.5 & 0.020 & 92.5 & 0.020 & 92.9 & 0.020 & 92.5 \\
\hline 8 & BHT & 0.057 & 77.8 & 0.057 & 77.8 & 0.057 & 77.8 & 0.057 & 77.8 \\
\hline 9 & Blank & 0.257 & 0.0 & 0.257 & 0.0 & 0.257 & 0.0 & 0.257 & 0.0 \\
\hline
\end{tabular}

Values are mean \pm SD of triplicate experiment. Small alphabets in superscript within the rows indicate significant different among the solvents tested. 


\section{REFERENCES}

1. B.B. Surjadinata and L. Cisneros-Zevallos, J. Food Sci., 68, 2735 (2003).

2. A. Mann, A. Banso and L.C. Clifford, J. Health Res., 10, 34 (2008).

3. B. Mahesh and S. Satish, World J. Agric. Sci., 4, 839 (2008).

4. W. Zheng and S.Y. Wang, J. Agric. Food Chem., 49, 5165 (2001).

5. R. Govindarajan, M. Vijayakumar, M. Singh, Ch.V. Rao, A. Shirwaikar, A.K.S. Rawat, P. Pushpangadan, J. Ethnopharmacol., 106, 57 (2006).

6. F. Biglari, A.F.M. AlKarkhi and A.M. Easa, Food Chem., 107, 1636 (2008).

7. A. Jabeen, M.A. Khan, M. Ahmad, M. Zafar and F. Ahmad, Afr. J. Biotechnol., 8, 763 (2009).

8. W. Charles and J.E. Hagen, Am. J. Bot., 53, 46 (1966).

9. B.A. Bohm and G.H.N. Towers, Can. J. Bot., 40, 677 (1962).

10. P. Panichayupakaranant, H. Noguchi and W. De-Eknamkul, Planta Med., 64, 774 (1998).

11. A. Hasan and M.N. Tahir, Turk. J. Chem., 29, 65 (2005).

12. N. Anwer, M.A. Waqar, M. Iqbal, M. Mushtaq and A. Sobia, Int. Food Res. J., 20, 99 (2013).
13. A. Sofowara, Spectrum Books Ltd., Ibadan, Nigeria, p. 289 (1993).

14. M.A. Iyengar, Study of Crude Drugs, Manipal Power Press, Manipal, India, edn. 8, p. 102 (1995).

15. T.S. Roopashree, D. Raman, R.R.H. Shobha and C. Narendra, Int. J. Appl. Res. Nat. Prod., 3, 20 (2008).

16. A.A. Siddiqui and M. Ali, Practical Pharmaceutical Chemistry, CBS Publishers and Distributors, New Delhi, edn. 2, p. 126 (1997).

17. S. Lee, S. Suh and S. Kim, Neurosci. Lett., 287, 191 (1999).

18. B. Sultana, F. Anwar and R. Przybylski, Food Chem., 104, 1106 (2007).

19. The Clinical Laboratory Standards Institute, J. Clin. Microbiol., 45, 2758 (2007).

20. H. Wang, X.D. Gao, G.C. Zhou, L. Cai and W.B. Yao, Food Chem., 98, 317 (2006)

21. H.P. Rupasinghe, C.J. Jackson, V. Poysa, C. Di-Berado, J.D. Bewley and J. Jenkinson, J. Agric. Food Chem., 51, 5888 (2005).

22. M. Sayyah, N. Hadidi and M. Kamalinejad, J. Ethnopharmacol., 92, 325 (2004).

23. M.S. Moghadam, S. Maleki, E. Darabpour, H. Motamedi, S. Mansour and S. Nejad, Asian Pac. J. Trop. Med., 3, 262 (2010). 